\title{
Staircase-free acoustic sources for grid-based models of wave propagation
}

\author{
Elliott S. Wise*, James L. B. Robertson, Ben T. Cox, Bradley E. Treeby \\ Department of Medical Physics and Biomedical Engineering, University College London, United Kingdom \\ *elliott.wise.14@ucl.ac.uk
}

\begin{abstract}
The k-Wave MATLAB toolbox is widely used to conduct medical ultrasound simulations. It uses a Fourier collocation method to numerically solve the governing model equations, and introduces sources by adding acoustic pressure at points on an orthogonal grid. This approach introduces two errors when sources don't exactly align with the grid. These are phase errors arising from shifting source points to nearby grid nodes, and amplitude errors arising from an angular dependence in the density of source points. These two errors are collectively referred to as 'staircasing'. Staircasing errors can be overcome by considering the band-limited representation of sources that arises from the use of a Fourier collocation method. To do so, sources are discretised by convolving a band-limited point source with the desired source geometry. To validate this approach, a comparison is made with current $k$-Wave source algorithms and with the FOCUS ultrasound simulation code. The new sources are shown to eliminate staircasing errors.
\end{abstract}

Index Terms-acoustic waves, numerical simulation, Fourier series, function approximation

\section{INTRODUCTION}

Medical ultrasound simulations are used in a range of applications, including photoacoustics [1], focussed ultrasound therapies [2], [3], and ultrasound computed tomography [4]. In a research setting, the k-Wave MATLAB toolbox [5] is widely used to perform these simulations. A key algorithm that the toolbox uses is the Fourier collocation method for computing spatial derivatives. This requires the simulated domain to be discretised onto an orthogonal grid of nodes. Many factors influence the necessary spacing of nodes in this grid. The Nyquist limit states that there should be at least two grid points for the shortest acoustic wavelength of interest. However, in practice, other factors give more stringent requirements. One such factor is staircasing. This refers to the fact that continuously defined acoustic sources and media need to be sampled on the computational grid, thus producing a pixelated, or voxelised representation of the desired simulation. The effect of this in practice was recently examined for transcranial focussed ultrasound simulations [3]. Here, it was found that at least 20 points per wavelength were required for less than $10 \%$ error in peak focal intensity. In the present work, staircasing of acoustic sources is considered, and an algorithm is presented that eliminates source staircasing effects.

The authors would like to thank the EPSRC for funding this work.

\section{STAIRCASING ERRORS}

\section{A. The band-limited interpolant}

Fourier collocation methods represent a function using a weighted sum of complex exponentials. For a $d$-dimensional function $f$, this sum is given by

$$
f(\mathbf{x})=\sum_{\mathbf{k}} a(\mathbf{k}) e^{i \mathbf{k} \cdot \mathbf{x}},
$$

where $\mathbf{x} \in \mathbb{R}^{d}$ is the spatial coordinate vector, $\mathbf{k} \in \mathbb{Z}^{d}$ are the corresponding wavenumbers, and $a$ are the basis function weights. In practice, finite computational resources require these sums to be truncated, or band-limited, such that $\left|k_{j}\right| \leq k_{\max }=\pi / \Delta x_{j}$ for each dimension $j$. Here, $\Delta x_{j}$ is the grid node spacing.

Conventionally, source terms are introduced into Fourier spectral methods as a sum of point sources located on the grid nodes. However, introducing an exact point source, or delta function, is problematic due to band-limiting. Considering one-dimensional simulations, a delta function centred on the origin of the coordinate system has a Fourier transform which is constant for all frequencies. Band-limiting thus produces a function whose Fourier transform is a rectangle with a width that corresponds to the maximum supported frequency. This is known as a periodic sinc function [6]

$$
b(x)=\frac{1}{N} \cot \left(\frac{\pi x}{N \Delta x}\right) \sin \left(\frac{\pi x}{\Delta x}\right),
$$

where $N$ is the number of grid nodes (the formula above is for even $N$, but a similar function exists for odd $N$ ). An illustration is given in Fig. 1. When sampled on the grid, this function is one at the origin (assuming a node at $x=0$ ), and zero everywhere else. A similar argument holds in multiple dimensions, with band-limited multi-dimensional point sources being a product of periodic sinc functions in each dimension. This introduces the concept of a band-limited interpolant: given a discretised, periodic function that has been sampled on an orthogonal grid, the band-limited interpolant is the sum of periodic sinc functions shifted and scaled to each of the grid values.

Due to the convention of placing point sources on grid nodes only, staircasing errors arise. These have two causes. The first is undersampling. By only placing point sources on grid nodes, sources which are not parallel to the coordinate axes have a different spacing relative to those which are parallel. This causes a reduction in the amplitude of the source wave. 

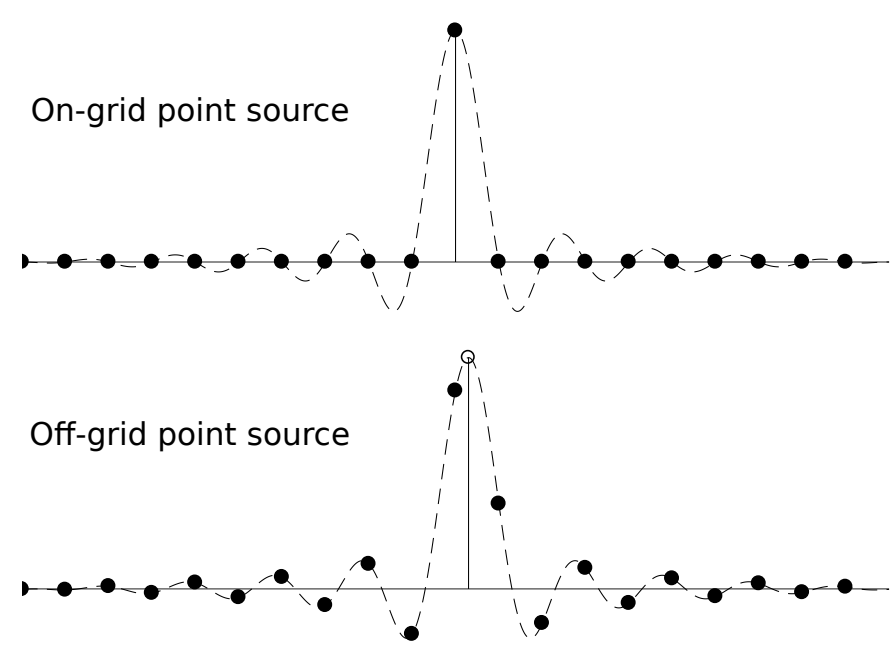

Fig. 1. Band-limited representations of point-sources. A vertical stem indicates the desired point source location, and black discs indicate source values on the grid. If the source aligns with the grid, its band-limited interpolant will be equal to one at that grid node, and zero at all other grid nodes. If it is unaligned, the band-limited interpolant becomes evident in its samples.

Undersampling errors can be reduced by scaling the source points based on the average neighbour distance [7], but this is computationally costly and does nothing to address the second cause of staircasing error, which is misalignment. This occurs because a continuously defined source might not pass through grid nodes precisely, and so the convention is to place point sources at nearby grid nodes. This creates unwanted phase offsets in the resulting waveforms.

\section{B. A rotating line source}

To illustrate staircasing errors, a rotating line source is considered. A Gaussian pulse is produced and propagated past a sensor at the coordinate system's origin. The source is placed so that it lies exactly on the computational grid when it is parallel to the coordinate axes. The source is introduced on the grid nodes that are nearest to the desired line, and is simply connected (meaning no grid node has more than two neighbours). Figure 2 depicts the resulting acoustic field at two times for a $45^{\circ}$ orientation angle, along with the sensor data gathered from these simulations. Relative to the aligned case $\left(\theta=0^{\circ}\right)$, the amplitude of the sensor data can be seen to reduce by approximately $30 \%$ at $45^{\circ}$, corresponding to undersampling by a factor of $1 / \sqrt{2}$, as expected. The sensor data also shows a phase offset that varies with the source angle, but no obvious pattern is evident.

\section{THE HUYGENS-FRESNEL PRINCIPLE AND BAND-LIMITING}

In §II-A, the band-limited interpolant was considered to have arisen from a discretised source function whose samples lay on the computational grid. To address staircasing, a bandlimited interpolant needs to be defined for a continuous source function. Such a source can be viewed from the perspective of the Huygens-Fresnel principle, which states that a continuous
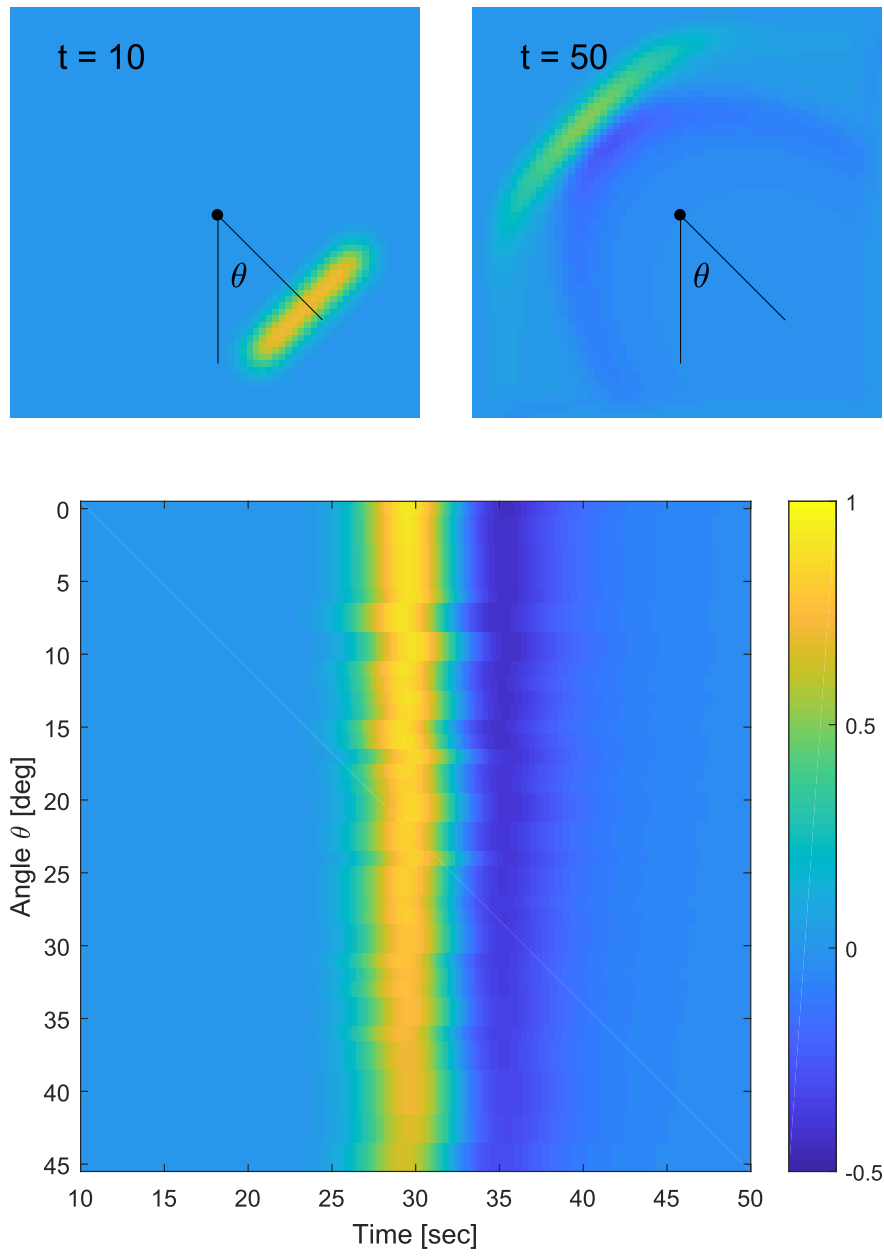

Fig. 2. A rotating on-grid line source emitting a Gaussian pulse. (Top) Snapshots of the acoustic field for $\theta=45^{\circ}$. The acoustic sensor is indicated by a black disc. (Bottom) Magnitude of the acoustic field at the sensor over time as a function of the source's orientation angle $\theta$. Staircasing errors are evident in the amplitude variations and time-offsets in the sensor data.

wave front is equivalent to an infinite number of point sources. Hence, a source function $f$ that is equal to one over the source, and zero everywhere else is equivalent to an infinite sum of shifted delta functions. To band-limit this, each point source is replaced with a periodic sinc function, achieved by a convolution

$$
f_{B L}(\mathbf{x})=\int_{\Omega} b\left(\mathbf{x}-\mathbf{x}^{\prime}\right) f\left(\mathbf{x}^{\prime}\right) d \mathbf{x}^{\prime},
$$

where $\Omega$ is the periodic computational domain. The bandlimited source $f_{B L}$ can then be discretised by sampling it on the computational grid nodes $\mathbf{x}_{n}$

$$
f_{n}=\left.f_{B L}(\mathbf{x})\right|_{\mathbf{x}=\mathbf{x}_{n}} .
$$

The effect of placing point sources off-grid can be seen in Fig. 1, with the periodic sinc function $b$ evident in the on-grid samples.

The convolution in (1) can be achieved in a computationally efficient manner by noting that introducing an infinite number of point sources is not numerically necessary. Band-limiting 
spreads the introduced acoustic pressure such that only a small degree of upsampling is needed relative to the computational grid. Figure 4 depicts four band-limited interpolants that illustrate the effect of band-limiting in different source discretisations. The left-most two plots show a conventional line source that has been discretised using point sources that align with grid nodes near the true continuous line. It can be seen that the resulting BLI oscillates around this line, and does not produce a smooth, continuous distribution of pressure. The remaining plots depict off-grid sources that result from discretising the convolution in (1) at half, twice, and ten-times the sampling rate of the computational grid. The constituent sinc functions can all be seen to lie exactly on the continuous source line. For the undersampled case, they are too far separated to approximate a constant distribution of pressure. However, convergence is rapid as the sampling rate increases, with no visual distinction evident between sources generated at upsampling rates of twice and ten-times the grid resolution. This indicates that only a small degree of upsampling is necessary for numerical accuracy.

\section{NUMERICAL EXAMPLES}

\section{A. A rotating line source}

To validate the convolution approach described above, the line-source experiment from $\S$ II-B was repeated with an offgrid source that was band-limited using periodic sinc functions sampled at a rate which is twice that of the computational grid. Figure 3 depicts these results. As the angle of the source changes, the amplitude and phase of the sensor data are nearly unaffected, unlike in Fig. 2. When compared with the aligned case $\left(\theta=0^{\circ}\right)$, the maximum variation in the sensor data is approximately $1.3 \%$ of the peak pressure at the sensor. These errors are likely the result of an orientation-dependence in the Gibbs phenomenon, since different numbers of spatial wavenumbers are supported in different angular directions.

\section{B. Comparison with FOCUS for a bowl source}

To illustrate the potential of off-grid sources in a more complex scenario, the acoustic field from a three-dimensional bowl source was simulated using k-Wave. A reference solution was provided by the FOCUS simulation toolbox [8], [9], which uses the fast near-field method to compute the acoustic field from a continuously defined bowl. It can be seen in Fig. 5 that the near-field contains significant errors when an ongrid source is used with $\mathrm{k}$-Wave, whereas an off-grid source gives nearly perfect results with a computational grid that has been sampled at only three points per wavelength, close to the Nyquist limit of two points per wavelength. In this case, the off-grid approach produced a maximum error in the axial pressure that was 17 times lower than that produced by the conventional on-grid approach.

\section{CONCLusion}

A technique is presented for introducing source terms in Fourier collocation methods at points which are not aligned

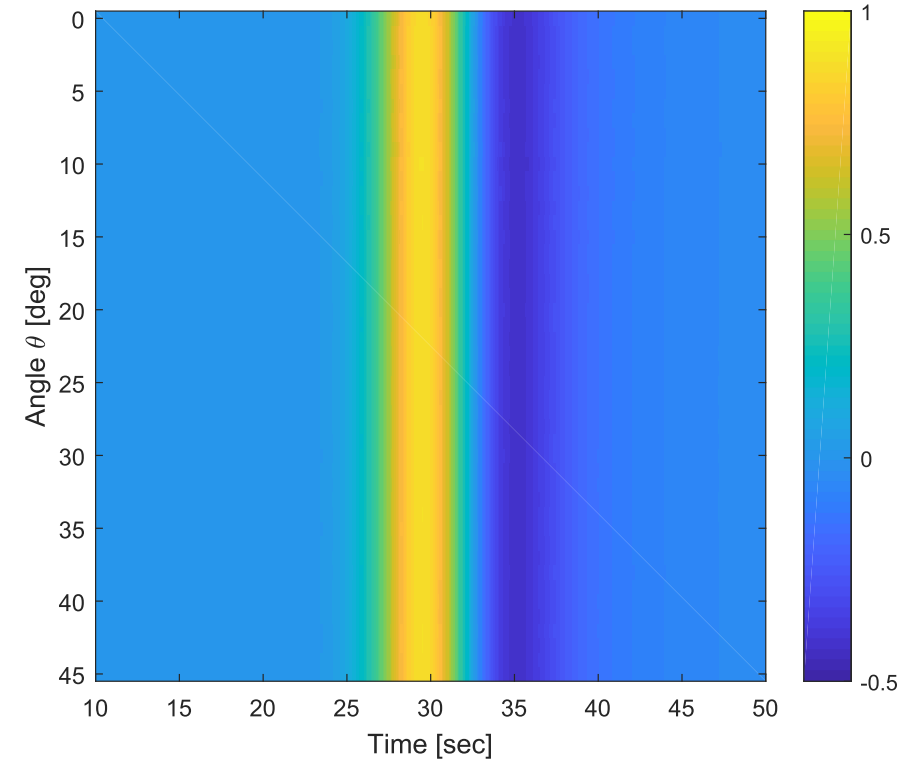

Fig. 3. The experiment depicted in Fig. 2 was repeated using an off-grid line source. This figure depicts the magnitude of the acoustic field at the sensor over time as a function of the source's orientation angle $\theta$. Staircasing errors have been eliminated.

with the computational grid. This eliminates staircasing errors, and gives a more faithful representation of continuously defined sources. Ongoing work will validate this technique further, and integrate it into the k-Wave MATLAB toolbox. Additionally, an investigation into using this technique for offgrid sensor discretisations is being undertaken, since the BLI corresponding to an off-grid sensor gives quadrature weights that interpolate and integrate the acoustic field over the sensor.

\section{REFERENCES}

[1] B. E. Treeby, J. Jaros, and B. T. Cox, "Advanced photoacoustic image reconstruction using the k-Wave toolbox," Proc. SPIE, vol. 9708, 2016.

[2] P. V. Yuldashev and V. A. Khokhlova, "Simulation of three-dimensional nonlinear fields of ultrasound therapeutic arrays," Acoust. Phys., vol. 57, no. 3, pp. 334-343, 2011.

[3] J. L. B. Robertson, B. T. Cox, J. Jaros, and B. E. Treeby, "Accurate simulation of transcranial ultrasound propagation for ultrasonic neuromodulation and stimulation," J. Acoust. Soc. Am., vol. 141, no. 3, pp. 1726-1738, 2017.

[4] M. Pérez-Liva, J. L. Herraiz, N. González-Salido, L. Medina-Valdés, J. Camacho, C. Fritch, and J. M. Udías, "Ultrasound computed tomography for quantitative breast imaging," in Global Medical Engineering Physics Exchanges/Pan American Health Care Exchanges (GMEPE/PAHCE), 2016, pp. 1-6.

[5] B. E. Treeby and B. T. Cox, "k-Wave: MATLAB toolbox for the simulation and reconstruction of photoacoustic wave fields," J. Biomed. Optic., vol. 15, no. 2, p. 021314, 2010.

[6] L. N. Trefethen, Spectral methods in MATLAB. Philadelphia: SIAM, 2000.

[7] E. Martin, Y. T. Ling, and B. E. Treeby, "Simulating Focused Ultrasound Transducers Using Discrete Sources on Regular Cartesian Grids," IEEE Trans. Ultrason., Ferroelect., Freq. Control, vol. 63, no. 10, pp. 15351542, 2016.

[8] R. J. McGough, "Rapid calculations of time-harmonic nearfield pressures produced by rectangular pistons," J. Acoust. Soc. Am., vol. 115, no. 5, pp. 1934-1941, 2004.

[9] D. Chen and R. J. McGough, "A 2D fast near-field method for calculating near-field pressures generated by apodized rectangular pistons," J. Acoust. Soc. Am., vol. 124, no. 3, pp. 1526-1537, 2008. 

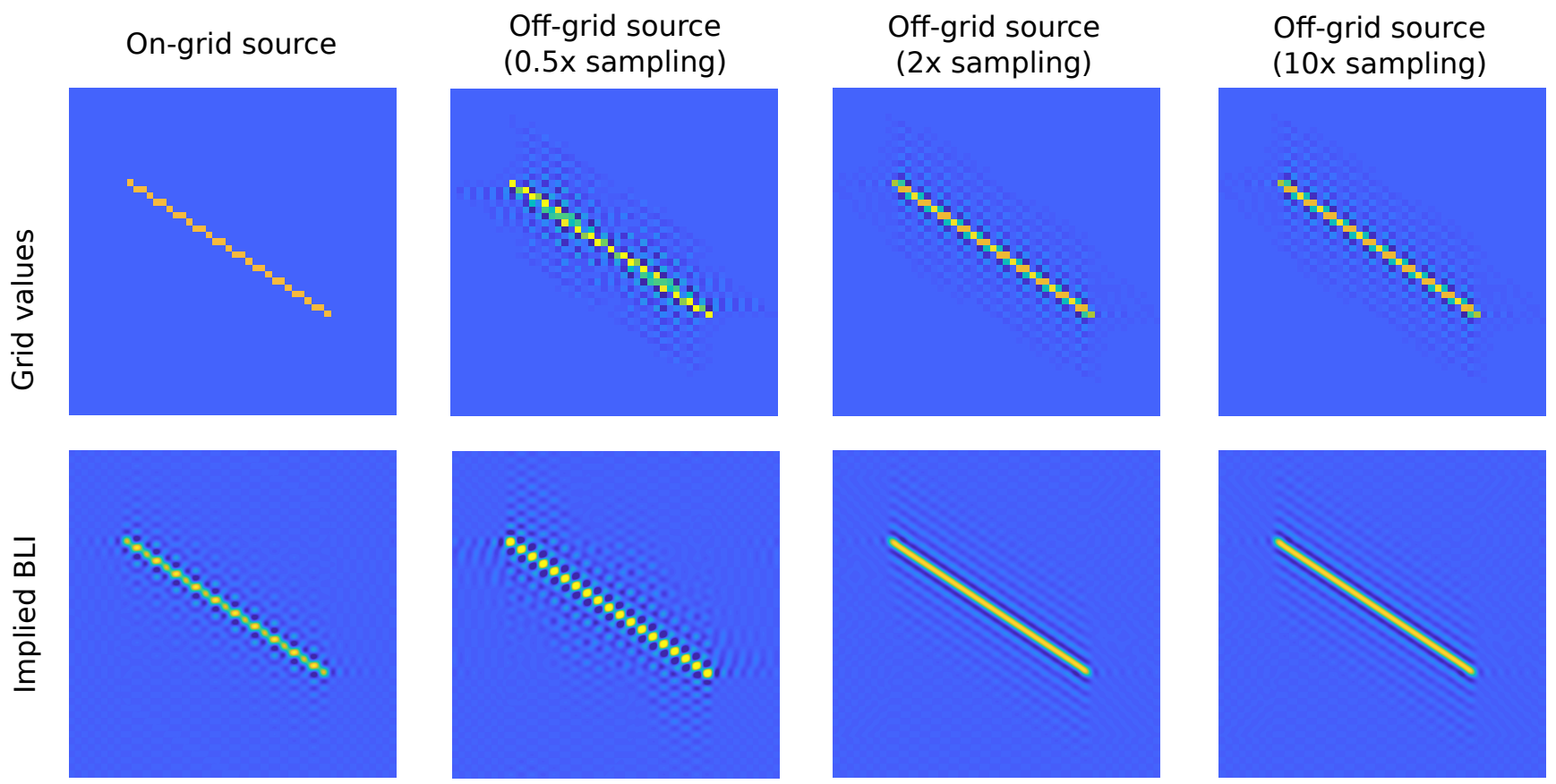

Fig. 4. Three different discretised/band-limited line sources. (Left-right) On-grid, undersampled $(0.5 \times)$ off-grid, oversampled $(2 \times)$ off-grid, and oversampled $(10 \times)$ off-grid sources. (Top) Source values on the computational grid and (bottom) implied band-limited interpolant (BLI). The sinc functions that comprise the on-grid source's BLI lie on either side of the desired source line. In contrast, the sinc functions that comprise the off-grid sources lie on the desired source line. The sinc functions that comprise the undersampled source's BLI are clearly separated, but oversampled off-grid sources quickly convergence on a smooth, continuous line. Note: Band-limited point sources decay quickly with distance. Hence, to reduce the memory requirements of this technique, source contributions are truncated once they decay below ten percent of their peak.

\section{Grid-Based Source}
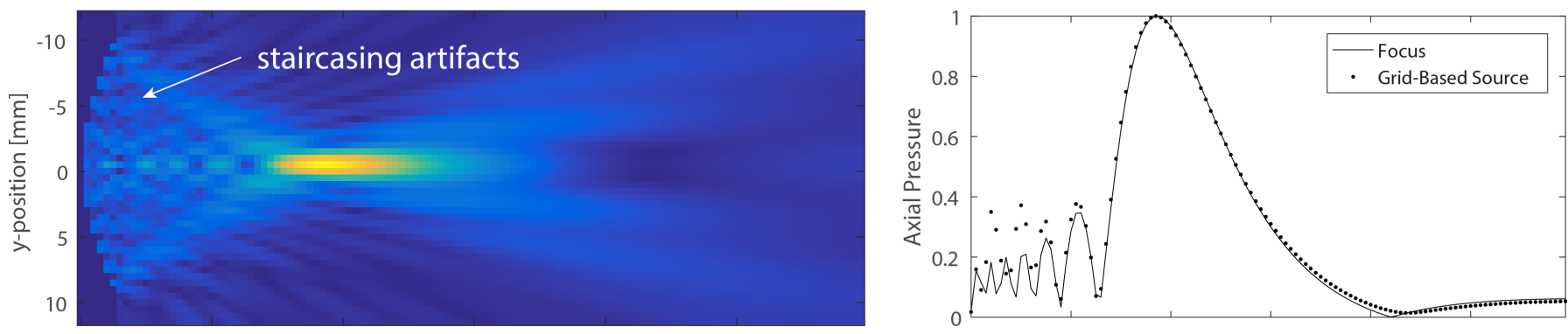

Off-Grid Source
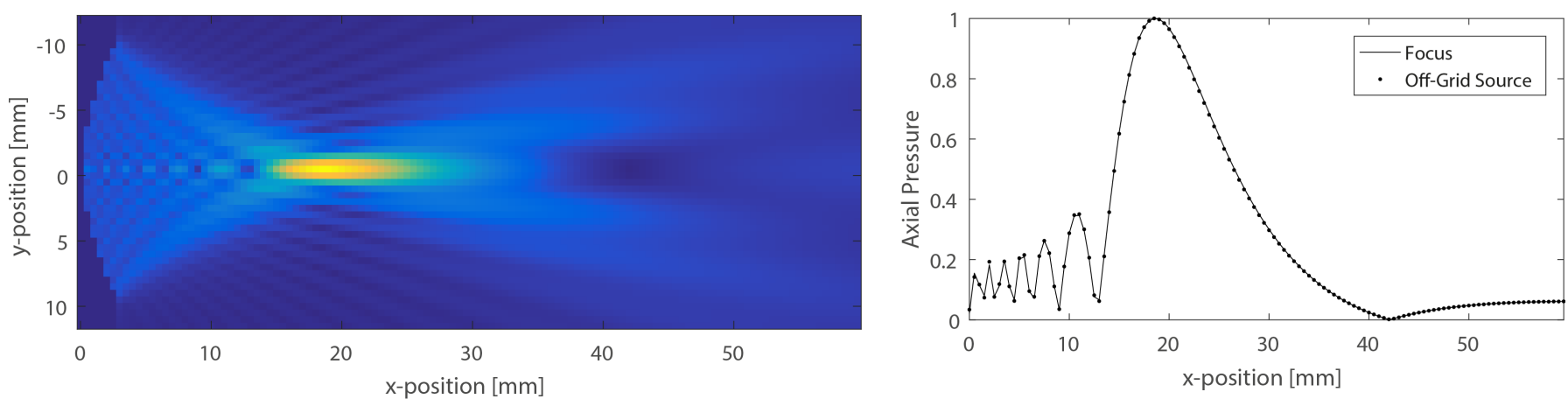

Fig. 5. A comparison between grid-based and off-grid bowl-source algorithms used in the k-Wave toolbox, with results from the FOCUS toolbox as a reference. The left plots depict the maximum steady-state pressure on a slice through the three-dimensional simulation. The right plots show the maximum axial pressure. Staircasing artefacts are particularly noticeable in the near-field of the grid-based source. 